respectively. Corresponding segmental CV were 35.2\%, 27.5\% and $33.5 \%$ (Abstract 098 table 2). The agreement between segmental perfusion and MPR from scans 1 and 2 are shown in Abstract 098 figure 1 . The reproducibility of left ventricular volumes and function was excellent (CV 4\%, 7.7\% and 4.6\% for end diastolic volume, endsystolic volume and ejection fraction respectively). There were no significant diurnal variations in perfusion or LV volumes and function.
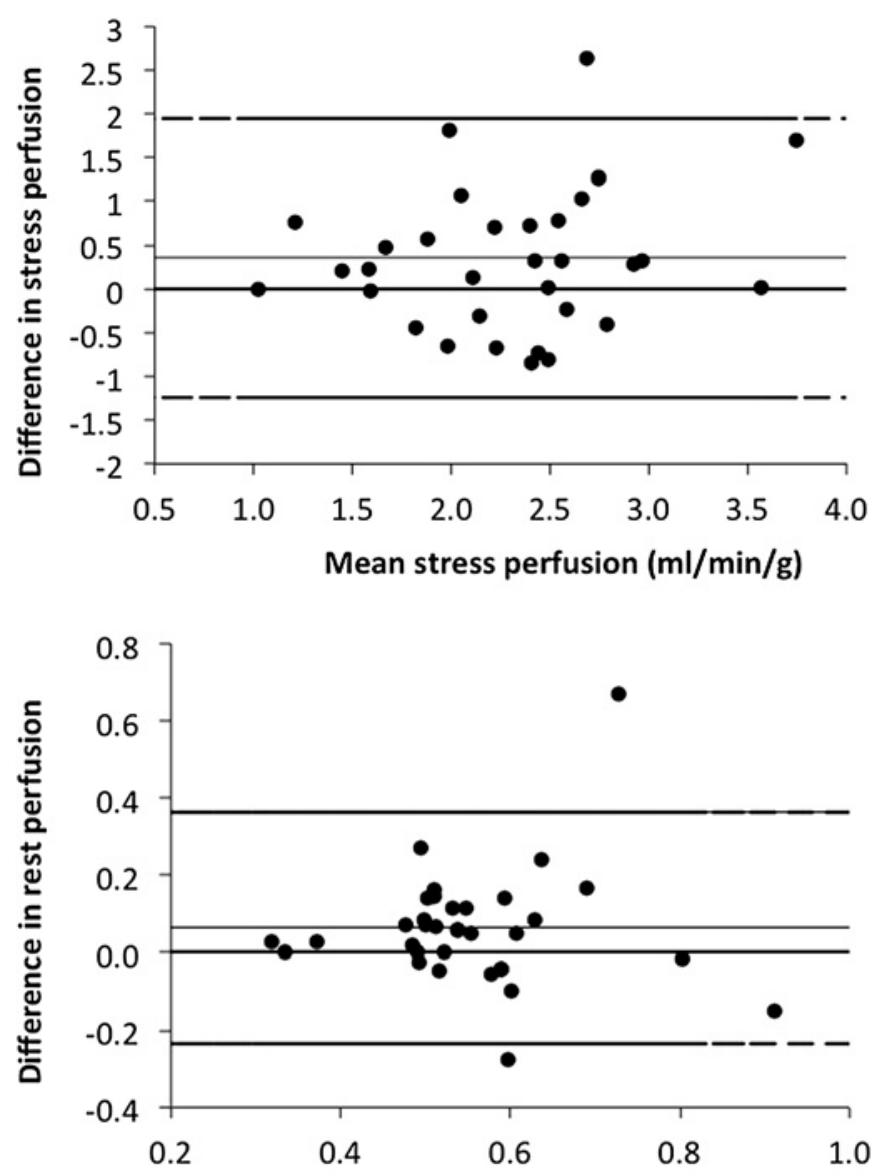

Mean rest perfusion $(\mathrm{ml} / \mathrm{min} / \mathrm{g})$

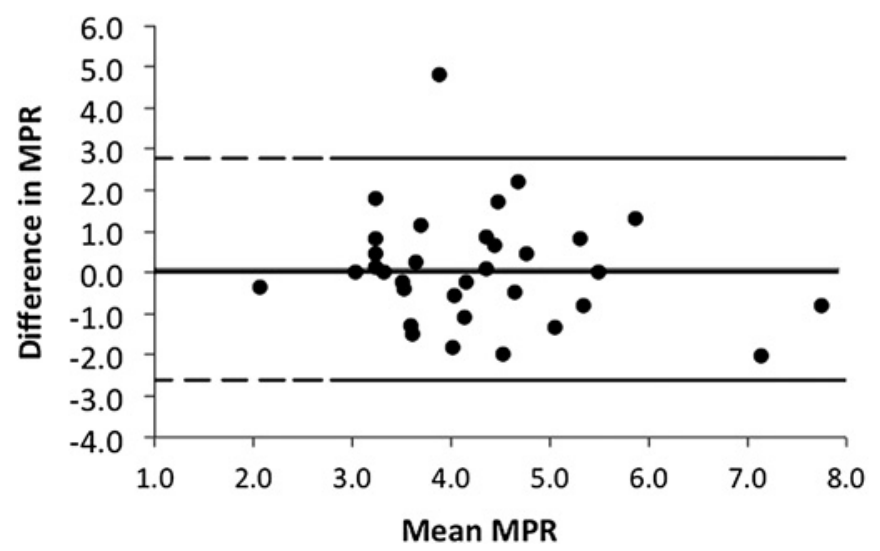

Abstract 098 Figure 1

Conclusions The inter-study reproducibility of quantitative myocardial perfusion is reasonable and best for global rest perfusion. No significant diurnal variation in perfusion was observed.
099 DYNAMIC THREE-DIMENSIONAL WHOLE HEART MAGNETIC RESONANCE MYOCARDIAL PERFUSION IMAGING: VALIDATION AGAINST THE DUKE JEOPARDY SCORE TO ASSESS MYOCARDIUM AT RISK

doi:10.1136/heartjnl-2012-301877b.99

R Jogiya, ${ }^{*}$ G Morton, K De Silva, D Perera, S Redwood, S Kozerke, E Nagel, S Plein. Kings College London, London, UK

Background Three-dimensional (3D) myocardial perfusion cardiovascular magnetic resonance (CMR) permits whole heart coverage and can establish an estimation of myocardium at risk and ischaemic burden. For invasive estimation of ischaemic burden, semi-quantitative angiographic scores including the Duke Jeopardy Score have clinical legitimacy as the magnitude of myocardium at risk due to severe coronary stenosis is associated with an adverse prognosis. The Duke Jeopardy score combines assessment of stenosis severity and location.

Objectives To determine the association between myocardium at risk defined by the Duke Jeopardy Score and 3D CMR perfusion imaging. Methods 53 patients referred for angiography underwent rest and adenosine stress $3 \mathrm{D}$ myocardial perfusion CMR at 3Tesla (3D turbo gradient echo, flip angle 15, TR $2.0 \mathrm{~ms} / \mathrm{TE} 1.0 \mathrm{~ms}$, 12 slices of $5 \mathrm{~mm}$ thickness, in-plane resolution $2.3 \times 2.3 \mathrm{~mm}^{2}, 10$-fold k-space and time k-t broad linear speed up technique acceleration with $k$ - $t$ principal component analysis). Volume of myocardial hypoperfusion was calculated by a blinded observer using with GTVolume software (GyroTools, Switzerland) with quantitative methods based upon adjusting the signal intensity threshold $>2$ SDs below the signal of remote myocardium. Volume of hypoperfusion was calculated by summation of the contiguous slices. Jeopardy score was calculated from the coronary angiograms to quantify the myocardium at risk. The coronary tree was divided into six segments of nearly equal myocardial perfusion (eg, left anterior descending artery, major diagonal branch, circumflex artery, major obtuse marginal branch artery, right coronary artery, and posterior descending artery). A score of 2 for each significant lesion was given. Vessels were analysed by a cardiologist blinded to CMR and clinical details and assigned a score ranging from 0 (no Jeopardy) to 12 (maximum Jeopardy). Results 53 patients were scanned with 159 coronary vessels anaylsed. The mean percentage volume of hypoperfusion on $3 \mathrm{D}$ CMR was $9.9 \%$ ( \pm 10.9 ). The mean Jeopardy Score was 4.0 ( \pm 3.9$)$. The mean percentage volume of hypoperfusion for Jeopardy scores

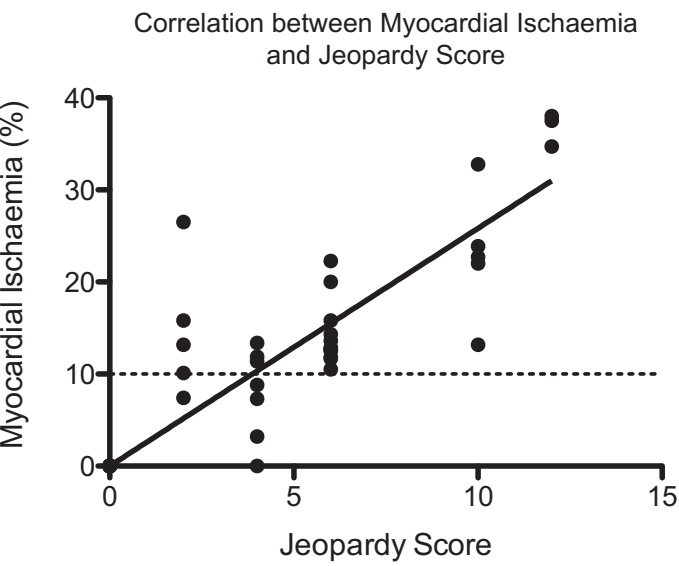

Abstract 099 Figure 1 Strong correlation between invasive measures of disease severity and ischaemic burden $(r=0.82)$. The dotted line represents the $10 \%$ threshold for which revascularisation may confer prognostic benefit over medical therapy alone. 
of $0,6,12$ were $0,13.1 \%$ and $36.7 \%$ respectively. Pearsons correlation coefficient showed a strong correlation ( $r=0.82,95 \%$ CI 0.70 to 0.89$)$ between the Jeopardy Score and volume of hypoperfusion on CMR $(\mathrm{p}<0.0001)$ (Abstract 099 figure 1).

Conclusion There is a strong correlation between myocardium at risk by invasive indices and volume of inducible ischaemia by dynamic 3D CMR whole heart perfusion imaging. 3D CMR perfusion imaging offers a non-invasive alternative method of detecting ischaemic burden and myocardium at risk for the purpose of serial studies, guiding revascularisation and risk stratification.

\section{DIAGNOSTIC ACCURACY OF HIGH DEFINITION COMPUTED TOMOGRAPHIC CORONARY ANGIOGRAPHY COMPARED TO INVASIVE CORONARY ANGIOGRAPHY IN THE ASSESSMENT OF PATIENTS WITH HIGH PRE-TEST PROBABILITY OF OR ESTABLISHED CORONARY ARTERY DISEASE}

doi:10.1136/heartjnl-2012-301877b.100

S Iyengar, ${ }^{*} 0$ Gosling, V Raju, G Morgan-Hughes, C A Roobottom. Plymouth Hospital NHS Trust, Plymouth, UK

Background 64-slice Computed Tomographic Coronary Angiography (CTCA) has high negative predictive value in assessment of patients with low-risk of coronary disease (CAD). However, it has a low specificity in assessment of patients with high pre-test probability of or established $\mathrm{CAD}$, due to limited spatial resolution and blooming artefact from coronary artery calcium. Recently published NICE guidelines recommend the use of conventional CTCA for the assessment of patients with low pre-test probability of CAD, but not for patients with high pre-test probability of CAD or those with calcified coronary atheromatous disease (Agatston Calcium score >400). High-definition CT (HDCT) combined with the use of iterative reconstruction (ASIR), aims to address the shortcoming of conventional CT technology by improving spatial resolution and reducing calcium blooming artefact, without increasing ionising radiation exposure

Methods Patients with high pre-test probability of and established $\mathrm{CAD}$, were prospectively enrolled in our HD-CTCA accuracy trial. We present the interim results of our 50 consecutive patients who underwent HD-CTCA following invasive coronary angiography (ICA) for the assessment of coronary disease. HD-CTCA was conducted on all patients within 30 days of ICA. Anonymised ICA and HD-CTCA studies were evaluated separately and results compared with ICA as the reference standard.

Results All HD-CTCA studies were acquired using prospective gating, $100 \mathrm{kV}$ tube voltage and optimum radiation dose reduction strategies and images were reconstructed using 50\% ASIR. The male: female ratio was 37:13 and the median (IOR) age, BMI and Agatston Calcium Score of patients at the time of scanning were $67.5(60-76.5)$ years, $26.5(24.4-28.6) \mathrm{kg} / \mathrm{m}^{2}$ and $708(293-1615)$ respectively. The median (IOR) radiation dose was 151 (131-275) $\mathrm{mGy} . \mathrm{cm}$, representing effective doses of $4.2(3.7-7.7) \mathrm{mSv}$ using a cardiac specific conversion factor $(0.028 / \mathrm{cm})$. All coronary segments visualised on ICA were demonstrated on HD-CTCA. Of the 726 coronary segments evaluated on HD-CTCA, 96.4\% were of excellent, $2.8 \%$ moderate and $0.8 \%$ poor diagnostic quality. Compared to ICA, HD-CTCA had sensitivity and specificity of $97 \%$ (95\% CI $81 \%$ to $100 \%$ ) and $95 \%$ (95\% CI $72 \%$ to $99 \%$ ) on a per-patient basis and sensitivity and specificity of $94 \%$ (95\% CI $87 \%$ to $980 \%$ ) and $98 \%$ ( $95 \%$ CI $97 \%$ to $99 \%$ ) on a per-coronary segment basis respectively. Conclusion Our interim results demonstrate that HD-CTCA has excellent accuracy compared to ICA in the assessment of patients with high pre-test probability of CAD or with established CAD and can be performed within acceptable radiation dose limits.

\section{INCORPORATION OF STRESS ECHOCARDIOGRAPHY INTO AN ACUTE CHEST PAIN SERVICE PROVIDES EXCELLENT FEASIBILITY, EARLY TRIAGING AND ACCURATE RISK STRATIFICATION OF PATIENTS WITH SUSPECTED ACUTE CORONARY SYNDROME BUT NON-DIAGNOSTIC ECG AND NORMAL 12-H TROPONIN}

doi:10.1136/heartjnl-2012-301877b.101

${ }^{1,2} \mathrm{~B}$ N Shah, ${ }^{*} \mathrm{G}$ Balaji, ${ }^{1} \mathrm{~A}$ Al Hajiri, ${ }^{1}$ I $S$ Ramzy, ${ }^{1} \mathrm{~S}$ Ahmadvazir, ${ }^{1,2} \mathrm{R}$ Senior. ${ }^{1}$ Northwick Park Hospital, London, UK; ${ }^{2}$ Royal Brompton Hospital, London, UK

Background Acute chest pain accounts for a substantial proportion of patients attending the Emergency Department (ED). Initial investigations are frequently inconclusive and many patients thus require admission for further risk stratification. We have previously demonstrated the clinical benefits and cost savings of stress echocardiography (SE) compared to stress ECG for risk stratification of patients admitted with suspected acute coronary syndrome (ACS) but normal ECG and negative 12-h troponin. However, the feasibilty of SE in routine clinical practice and its ability to predict hard cardiac events in this patient population is unknown.

Methods Consecutive patients admitted via the ED with chest pain and who underwent SE within 24 h of admission via our acute chest pain service were assessed for feasibility of SE, time to test and were followed-up for hard cardiac events (cardiac death and acute myocardial infarction-AMI).

Results Of 719 consecutive patients, 674 (94.6\%) had diagnostic images at SE and were followed-up over 26 months. The median time to test for all patients was 1 day and median in-hospital length of stay for those with normal SE was also 1 day. There were 17 hard events (14 cardiac deaths and 3 AMI). Annualised hard cardiac event rate in the normal SE group ( $\mathrm{n}=517,73.6 \%$ ) was $0.58 \%$ compared with $3.5 \%$ in the abnormal SE group $(p=0.002)$. Cox regression analysis revealed that among clinical, ECG and SE variables, only abnormal SE [ $p=0.001$, HR 4.02, 95\% CI 1.73 to 9.36] and advancing age (10-year increase) $[p=0.005$, HR $1.70,95 \%$ CI 1.18 to 2.44$]$ were independent predictors of hard events in the multivariate model Similarly, abnormal SE was also the strongest predictor of cardiac death [ $\mathrm{p}=0.001, \mathrm{HR} 4.52,95 \%$ CI 1.81 to 11.3$]$. At any stage during follow-up, an abnormal SE carried at least a fourfold increased risk of either cardiac death or any hard event over a normal SE result.

Conclusion This is the first study to show that the incorporation of SE into a clinical acute chest pain service has excellent feasibility, provides rapid assessment with early triaging and accurate risk stratification of patients with suspected ACS but non-diagnostic ECG and negative 12 -h troponin.

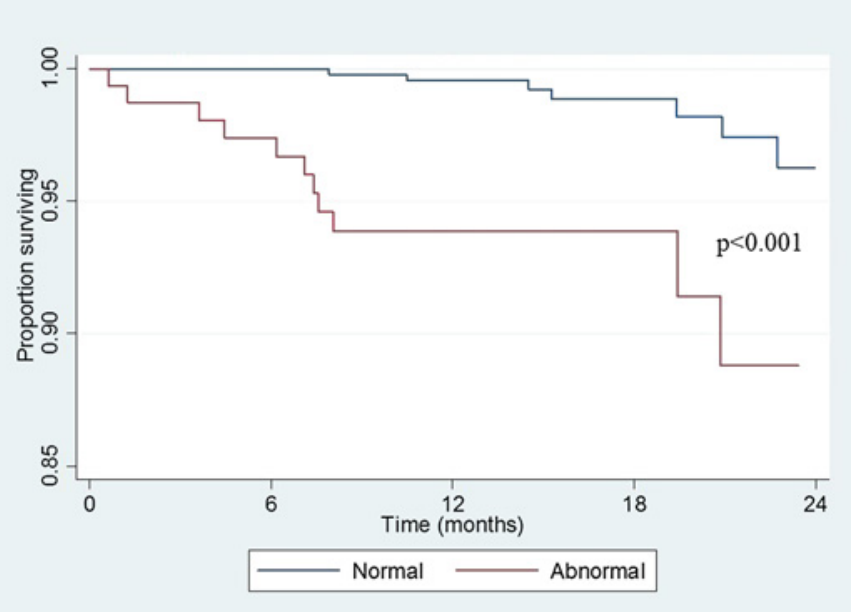

Abstract 101 Figure 1 Kaplan-Meier survival estimate of time to death. 